REVIEWS OF INFECTIOUS DISEASES • VOL. 9, SUPPLEMENT 5 • SEPTEMBER-OCTOBER 1987

(C) 1987 by The University of Chicago. All rights reserved. 0162-0886/87/0905-0045\$02.00

\title{
Glucocorticoid-Induced Impairment of Macrophage Antimicrobial Activity: Mechanisms and Dependence on the State of Activation
}

\author{
Andreas Schaffner and Thomas Schaffner
}

\author{
From the Department of Medicine, University of Zürich, \\ Zürich; and the Department of Pathology, \\ University of Bern, Bern, Switzerland
}

\begin{abstract}
Experimental observations indicate that tissue macrophages deployed in great numbers at critical anatomic sites such as the liver, spleen, and lung are major targets for glucocorticoids compromising natural resistance of the host. Therapeutic concentrations of glucocorticoids appear to prevent destruction of microorganisms ingested by macrophages without interfering with phagocytosis, phagolysosomal fusion, and/or secretion of reactive oxygen intermediates. These findings indicate that at the cellular level the glucocorticoid target should be sought for in the nonoxidative armature of the phagocyte and that nonoxidative killing systems of resident tissue macrophages play an important role in natural resistance to opportunistic pathogens. Glucocorticoids do not prevent lymphokine-induced activation of oxidative killing systems. Thus, lymphokines such as interferon- $\gamma$ can restore the microbicidal activity of macrophages functionally impaired by glucocorticoids. Counterbalance of the suppressive effect of glucocorticoids by lymphokines might only be possible, however, for pathogens susceptible to oxidative killing and not for microorganisms that are more resistant to reactive oxygen intermediates such as $A$ spergillus spores and Nocardia, opportunists that appear to be particularly associated with hypercortisolism.
\end{abstract}

From studies of populations of patients receiving glucocorticoid therapy [1-3] or suffering from endogenous hypercortisolism [4], it has become clear that glucocorticoids affect host resistance to a broad range of microorganisms. Analysis of large populations undergoing therapy with glucocorticoids suggests that a daily dose equal to or greater than the equivalent of $50-100 \mathrm{mg}$ of prednisone increases the patient's risk of developing an opportunistic infection $[2,3]$. Accordingly, a recent survey of infectious complications of endogenous hypercortisolism indicates that opportunistic infections develop when plasma cortisol levels are $0.4-1.8 \mu \mathrm{g} / \mathrm{ml}$ [4]. These concentrations of endogenous hormone correspond fairly well to the aforementioned dose of prednisone $[5,6]$.

Glucocorticoids have a bewildering array of effects on host defense systems, including effects on phagocytes and on cell-mediated and humoral immunity (reviewed in [7]), but the relevance of individual immunosuppressive mechanisms to the increased vulnerability of hypercortisolemic patients to infection has not been defined. Furthermore, it

This work was supported by grant 3.875 .85 of the Swiss National Science Foundation.

Please address requests for reprints to Dr. Andreas Schaffner, Medizinische Klinik, CH 13, CH-8091 Zürich, Switzerland. is not clear why infections with some pathogens, such as Aspergillus, Nocardia, and Pneumocystis, are particularly associated with hypercortisolism $[2,4]$.

Our interest in the mechanisms by which glucocorticoids damage host resistance arose from our studies on natural resistance to Aspergillus, which indicated that glucocorticoids rendered "resting" macrophages unable to confine and kill ingested spores [8]. Because glucocorticoid-mediated impairment of the antimicrobial function of tissue macrophages is critical for the immunosuppressive effect in aspergillosis, we studied the importance of such mechanisms for other infections. This paper outlines our recent experimental findings on how glucocorticoids impair antimicrobial activity of macrophages and how this impairment depends on the state of macrophage activation.

\section{Impairment of Conidiacidal Activity of Macrophages by Glucocorticoids}

Host resistance to conidia, the spores of Aspergillus, is largely independent of $\mathrm{T}$ lymphocytes, neutrophil granulocytes, and blood monocytes but is highly susceptible to damage by glucocorticoids (figure 1). Humoral immunity appears not to be of importance [8]. By exclusion, the candidate for the glucocorticoidsensitive target is the resident macrophage, a sugges- 
Figure 1. Clearance of inhaled conidia by normal, myelosuppressed, cortisone-treated, and athymic mice. Elimination of spores is not impaired by severe leukopenia or by the athymic state but is affected by immunosuppression with cortisone. Cortisone: animals were given six daily doses of cortisone (2.5 mg each dose) starting $36 \mathrm{~h}$ before challenge; mustard: animals were given three daily doses of nitrogen mustard (40 $\mathrm{\mu g}$ per dose), treatment leading to leukopenia ( $<200$ leukocytes and $<100$ neutrophils $/ \mathrm{mm}^{3}$ of blood) throughout the study; nu/nu: athymic (nude) BALB/c mice; nu/t: heterologous littermates. Each point represents the mean log cfu for three mice. Figure is modified from [8].

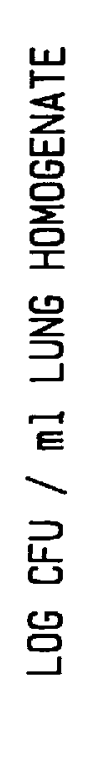

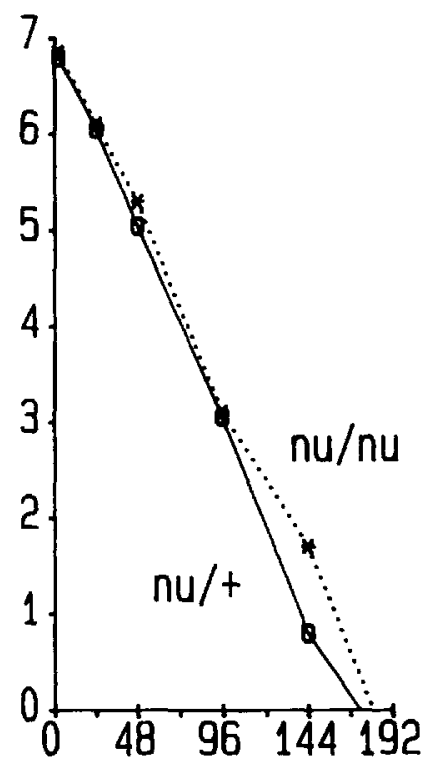

HOURS AFTER CHALLENGE tion made more than 20 years ago by Sidransky [9]. Impairment of the conidiacidal activity of mononuclear phagocytes by glucocorticoids results from a direct effect on the phagocyte (i.e., impairment is not mediated through systems modulating macrophage function), as readily demonstrated by exposing pure preparations of human mononuclear phagocytes to glucocorticoids $[8,10]$. This treatment results in a loss of anticonidial activity comparable to that seen in murine macrophages previously exposed to glucocorticoid in vivo or in vitro [8]. It is of note that impairment of the anticonidial activity occurs at pharmacologic concentrations (i.e., $10^{-8} M$ dexamethasone). This observation, together with the observations in mice, indicates that impaired killing (mainly by alveolar macrophages) of Aspergillus conidia is also operative in glucocorticoid-treated patients and thus contributes to the susceptibility of hypercortisolemic patients to aspergillosis (effects of cortisone on natural immunity against hyphae provided by neutrophil granulocytes are discussed in [8]).

\section{Impairment of Antibacterial Activity of Macrophages by Glucocorticoids}

Glucocorticoids are known to damage host resistance to intracellular bacteria [11, 12]. North [11] demonstrated that cortisone dramatically lowered resistance of mice to Listeria monocytogenes and provoked rapid proliferation of bacteria in the livers of glucocorticoid-treated animals. Because infiltration by small lymphoid mononuclear cells in the livers of glucocorticoid-treated animals was markedly reduced in comparison to that in control animals, and because the first studies showing an impaired antimicrobial activity of glucocorticoid-treated mononuclear phagocytes were not yet available [13, 14], North speculated that a suppression of the influx and/or the proliferation of lymphocytes was responsible for the immunosuppressive effect of glucocorticoids in murine listeriosis and hence that in this model lymphocytes were the cortisone target.

Our observation that glucocorticoids affect macrophages in the aspergillosis model and the fact that the glucocorticoid effect in North's experiments occurred before $\mathrm{T}$ cell-mediated control of listeriosis was apparent in control animals made us curious about whether mononuclear phagocytes rather than $\mathrm{T}$ cells were the target for the immunosuppressive effect of glucocorticoids in the murine listeriosis model. Indeed, when we compared the effects of cortisone in normal and athymic mice, we found that cortisone had virtually the same effect on the course of listeriosis in animals with functional $\mathrm{T}$ lymphocytes as in those without (figure 2). These experiments thus show that $\mathrm{T}$ lymphocytes cannot be the 


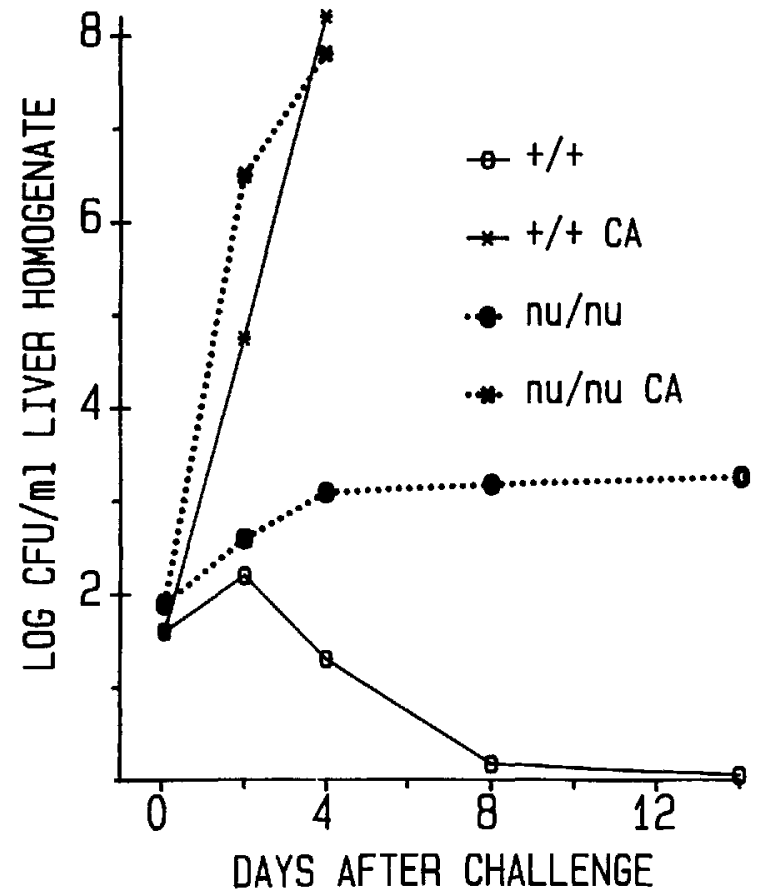

Figure 2. Comparison of the effect of cortisone acetate (CA) on listeriosis in athymic (nude) BALB/c mice and in their normal littermates. Cortisone was given at a daily dosage of $1 \mathrm{mg}$ per animal, starting 2 days before challenge and continuing until death, which invariably occurred within 6 days in animals receiving the drug. The challenge dose was $700 \mathrm{cfu}$ of a wild-type strain of Listeria monocytogenes in thymus-bearing mice $(+/+)$, and 2,800 $\mathrm{cfu}$ in nude mice (nu/nu), to obtain an initial bacterial count in the liver comparable to that in control mice. Each point represents the mean of $\log \mathrm{cfu}$ for four to six animals. Figure is modified from [15].

critical target for the immunosuppressive effect of glucocorticoids in this model of listeriosis and suggest that, as in aspergillosis, resistance provided by macrophages is affected by the steroid.

If host resistance provided by mononuclear phagocytes is the main target for the immunosuppressive effect of glucocorticoids in murine listeriosis, the question arises whether the ability of mononuclear phagocytes to accumulate at infectious sites is impaired [16] or, as in the aspergillosis model, the activity of resident macrophages is damaged. Eventually, of course, both mechanisms could contribute to the immunosuppressive effect of glucocorticoids. It is of note that in the listeriosis model $>90 \%$ of the organisms injected iv are cleared rapidly by the reticulohistiocytic system; $>90 \%$ of the challenge dose is recoverable from the liver and spleen within
10 min of injection [17] (figures 3 and 4). This finding is strong evidence for the localization of early listerial infection in resident tissue macrophages. Circumstantial evidence indicates that accumulation of blood-derived mononuclear phagocytes in listeric livers is critical for the control of listeriosis in this organ $[18,19]$. Nevertheless, our observations indicate that glucocorticoids interfere primarily with the natural resistance provided by the resident macrophages that first encounter the bacteria. When we attempted to duplicate the ef fect of glucocorticoids on phagocyte recruitment by ablation of the bone marrow with cytotoxic drugs, which rendered the mice severely leukopenic and unable to mount a cellular inflammatory response, the course of listeriosis was unaffected (figures 3 and 5). This finding indicates that accumulation of bone marrow-derived phagocytes is not important for control of listeriosis during the first $48 \mathrm{~h}$ after infection, the exact time period during which most of the immunosuppressive effect of glucocorticoids is operative (figure 2).

Because antibody and other humoral factors do not play important roles in murine listeriosis[17], these findings again point to the antimicrobial activity of tissue macrophages as the critical target for the immunosuppressive effect of glucocorticoids in the listeriosis model of North. Again, when pure preparations of mononuclear phagocytes are exposed to pharmacologic concentrations of glucocorticoids in vitro, the phagocytes lose their listericidal activity (figure 6) without undergoing any apparent nonspecific damage, as evidenced by parallel studies of viability, phagocytic rate, and secretion of lysozyme [10]. In agreement with these in vitro observations on human blood-derived phagocytes are the findings that glucocorticoids administered in vivo impair the capacity of phagocytes to reduce the inoculum and that tissue macrophages such as Kupffer cells fail to control early bacterial growth (figures 3 and 4). Furthermore, experiments with Salmonella typhimurium and Nocardia asteroides suggest that glucocorticoid-induced damage to the antimicrobial activity of macrophages is not limited to their activity against Aspergillus and Listeria [10].

\section{Effect of Glucocorticoids on the Antimicrobial Activity of Activated Macrophages and on Macrophage Activation}

The studies of North on the effects of glucocorticoids on murine listeriosis showed that mice become 
Figure 3. Comparison of the course of early listeriosis in ICR mice immunosuppressed with nitrogen mustard (HN2) or cortisone acetate and in control mice. In spite of the high dose of $1.8 \times 10^{5}$ Listeria monocytogenes (strain EGD) organisms per animal, the initial reduction of the challenge dose and the early growth rate was impaired only by cortisone but not by nitrogen mustard (three doses of $50 \mu \mathrm{g}$ ). Fifteen minutes after iv injection of the bacteria, $88 \%-91 \%$ of the challenge dose was recovered from the liver. Bone marrow suppression by nitrogen mustard was monitored by blood counts ( 0 granulocytes $/ \mathrm{mm}^{3}$ of blood). Each point represents the mean value for five animals (percentage of initial count in panel A; $\log _{10} \mathrm{cfu} / \mathrm{ml}$ in panel B). Bars give SD. The methods used were those described in [15], except that the total volume of the liver homogenate in the experiment described above was $5 \mathrm{ml}$.
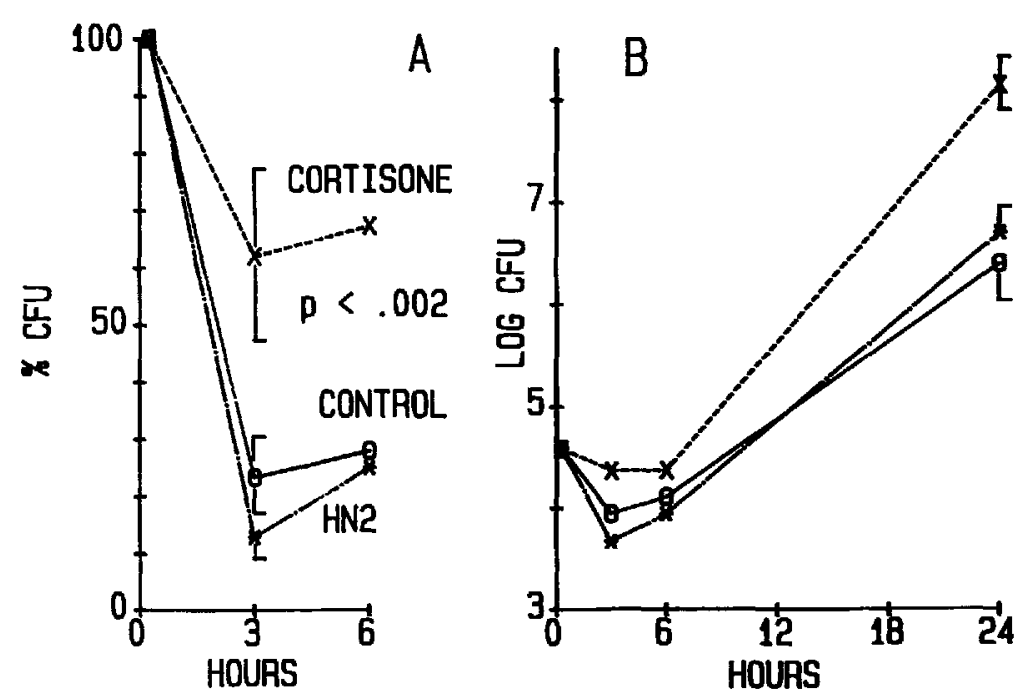

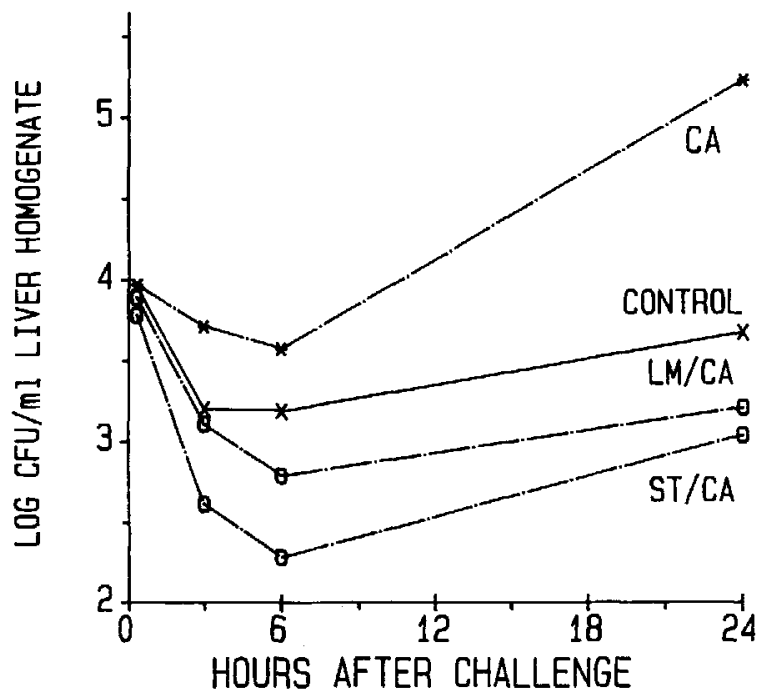

Figure 4. Effect of heterologous and homologous immunization with live bacteria on elimination of Listeria monocytogenes (strain EGD) from the liver. Fourteen days before challenge female ICR mice were given 0.01 of an $\mathrm{LD}_{\mathrm{so}}$ of L. monocytogenes strain EGD (LM) or Salmonella typhimurium (wild-type; ST) iv. Immunosuppression with cortisone acetate (CA) was started $36 \mathrm{~h}$ before iv challenge with $3.8 \times 10^{4} \mathrm{cfu}$ of $L$. monocytogenes. Values at each time point are mean $\log \mathrm{cfu}$ from the livers of five animals. CA: each animal received $2.5 \mathrm{mg} /$ day of cortisone acetate for 3 days. Note that the total volume of liver homogenate was $5 \mathrm{ml}$ and that $>85 \%$ of the inoculum was recovered from the liver $10 \mathrm{~min}$ after challenge (for methods see [15]). increasingly resistant to immunosuppression by glucocorticoids during the course of infection. He concluded that, while the developmental stage of immunity was highly sensitive to glucocorticoids, established immunity became increasingly less susceptible [11]. If we compare these observations to our finding that the antimicrobial activity of macrophages may be the critical target for the immunosuppressive effect of glucocorticoids for infection with intracellular pathogens such as Listeria, we would expect that the antilisterial capacity of "activated" macrophages is unaffected by glucocorticoids and that "adoptive glucocorticoid resistance" is nonspecific. Indeed, when mice are infected with a sublethal dose of S. typhimurium 14 days before treatment with glucocorticoid and are then challenged with Listeria, the immunosuppressive effect of cortisone on early listeriosis is no longer apparent. Heterologous immunization thus results in the elimination of Listeria from the livers of cortisonetreated animals that is even better than the elimination of the organisms from normal control animals (figure 4). This adoptive glucocorticoid resistance is not affected by concurrent exposure of immunized mice to glucocorticoid and cytotoxic drugs that abolish the mobilization of bone marrow-derived phagocytes (data not shown).

In these experiments it is of importance that the failure of glucocorticoid-treated animals to mobi- 


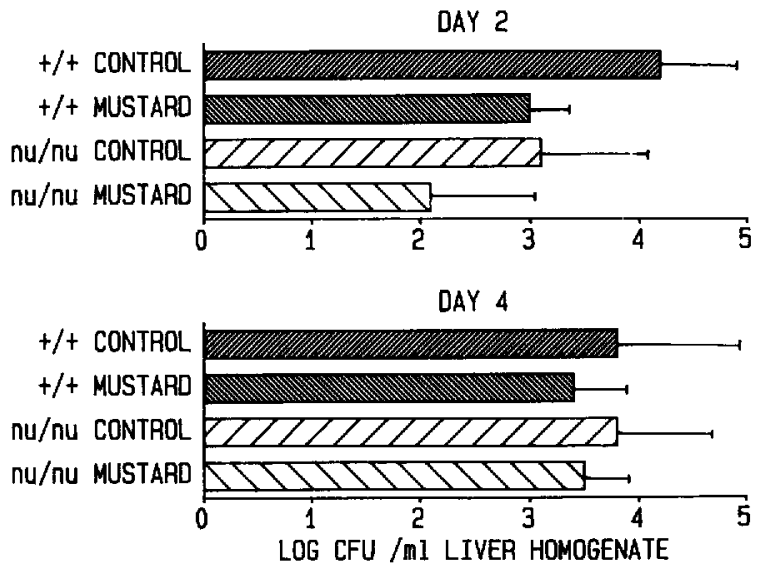

Figure 5. Inability of bone marrow suppression to affect the course of early listeriosis in normal $(t / t)$ and athymic (nu/nu) BALB/c mice. Ablation of bone marrow with nitrogen mustard, a treatment resulting in severe leukopenia and in a complete loss of the ability to mount a cellular inflammatory response to heat-killed bacteria in the peritoneal cavity, does not affect the course of listeriosis induced by a low challenge dose of a wild-type strain of Listeria monocytogenes over 4 days of infection. Each point represents the mean $\log$ cfu from four to six animals (figure is modified from [15]).

lize mononuclear phagocytes is not alleviated by prior immunization (figure 7). These observations prove that resistance to glucocorticoid that is induced by immunization with live bacteria does not reside in the antigen-specific afferent limb of cell-mediated immunity, i.e., in T cells. The findings further indicate that the effector cells, the macrophages resident in the liver before challenge, become resistant to cortisone after infection with Listeria or Salmonella. Accordingly, we found that the antilisterial activity of human blood-derived macrophages activated by interferon- $\gamma$ is completely unaffected by pharmacologic concentrations of dexamethasone in vitro [10]. Furthermore, glucocorticoids do not affect the activation of antilisterial activity by interferon- $\gamma$, even after macrophages are pretreated for prolonged periods with pharmacologic concentrations of dexamethasone [10] or exposed to suprapharmacologic concentrations of hydrocortisone succinate (figure 6). These observations are in agreement with those of Nash et al. [20] that hydrocortisone does not interfere with a lymphokine-mediated suppression of intracellular replication of Legionella pneumophila in human macrophages but is at variance with observations by Masur et al. [21] that crude lymphokine preparations do not activate mouse peritoneal mac- rophages against Toxoplasma gondii in the presence of high concentrations of hydrocortisone.

Although the antilisterial activity of dexamethasone-treated macrophages is restored by interferon$\gamma$, this lymphokine has no effect on the anticonidial activity of macrophages and thus does not correct for the dexamethasone effect on the anticonidial activity of human alveolar or blood-derived macrophages [10].

In accordance with the apparently opposing effects of dexamethasone and interferon- $\gamma$ on antilisterial activity of macrophages, activation of the antilisterial potential of the macrophage is not suppressed by the addition of pharmacologic concentrations of dexamethasone to mixed-cell systems composed of proliferating lymphocytes (stimulated by a mitogen or by an antigen) and macrophages, in spite of a marked suppression of lymphocyte

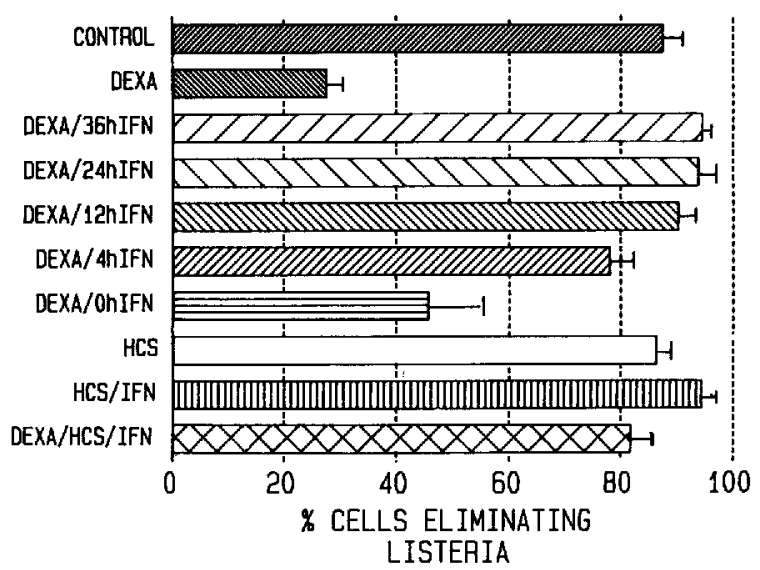

Figure 6. Effect of glucocorticoids and interferon- $\gamma$ on the elimination of Listeria monocytogenes ingested by human blood-derived macrophages cultured in vitro for $36 \mathrm{~h}$ before challenge. Values are the mean percentage $( \pm$ SD) of cells from quadruplicate wells that eliminated ingested bacteria during $8 \mathrm{~h}$ of incubation, as determined by enumeration of bacteria in May-Giemsa-Grünwald-stained monolayers (methods according to [10]). Supplements added to the macrophage cultures before challenge were as follows. DEXA: $2.5 \times 10^{-7} M$ dexamethasone for $36 \mathrm{~h}$; IFN: 120 units of human recombinant interferon- $\gamma / \mathrm{ml} \mathrm{0-36} \mathrm{h}$ before challenge; HCS: $100 \mu \mathrm{g}$ of hydrocortisone succinate added $18 \mathrm{~h}$ before challenge; HCS/IFN: $100 \mu \mathrm{g}$ of hydrocortisone succinate $18 \mathrm{~h}$ before challenge and 120 units of interferon- $\gamma 12 \mathrm{~h}$ before challenge. Note that interferon- $\gamma$ restored the listericidal activity of macrophages in the continuous $(36 \mathrm{~h})$ presence of dexamethasone; suprapharmacologic concentrations of hydrocortisone added to cultures for only $18 \mathrm{~h}$ had no effect on the antilisterial activity and did not inhibit the restoration by interferon- $\gamma$ of the antimicrobial function of dexamethasone-treated cells. 


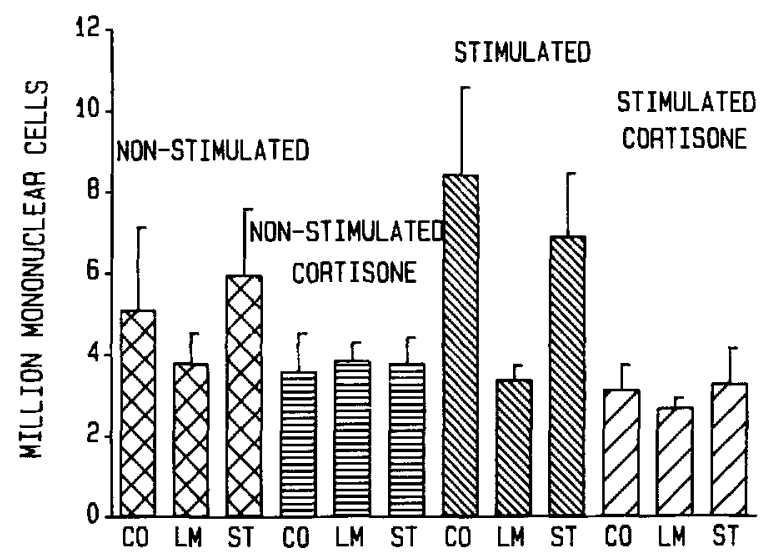

Figure 7. Effect of cortisone $(\mathrm{CO})$ and immunization with live bacteria (Listeria monocytogenes [LM] or Salmonella typhimurium [ST]) on an inflammatory mononuclear cellular response in the peritoneal cavity. Female ICR mice were immunized and given cortisone as outlined in figure 4. Twenty-four hours before mice were killed, an inflammatory cellular response was initiated by injecting them ip with $10^{9}$ heat-killed $L$. monocytogenes organisms $\left(120^{\circ} \mathrm{C}, 15 \mathrm{~min}\right)$ suspended in saline. Peritoneal cells were enumerated and differentiated as described [8]. Note that neither homologous (LM) nor heterologous (ST) immunization alleviated the suppression of the inflammatory cellular response to the stimulus. Results are for one typical experiment of two experiments.

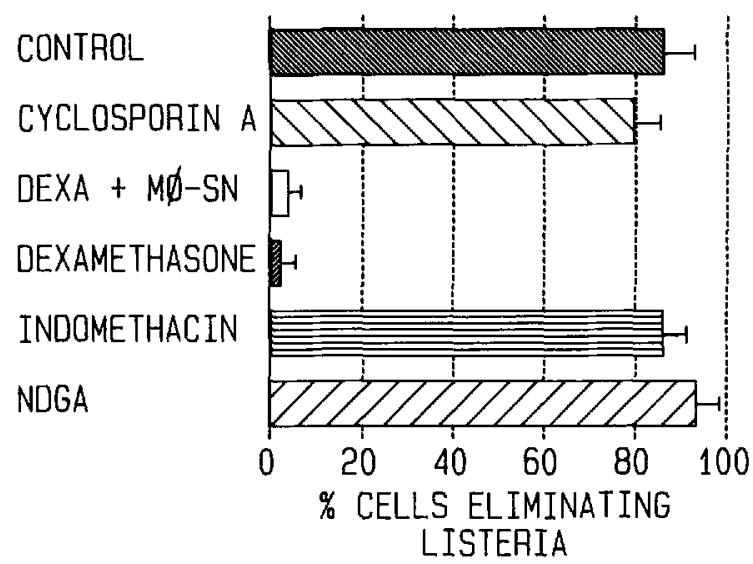

Figure 8. Effects of dexamethasone, inhibitors of leukotriene and prostaglandin synthesis, cyclosporin $A$, and macrophage supernatant on the elimination of Listeria monocytogenes from human blood-derived macrophages. Before challenge with $L$. monocytogenes, human mononuclear phagocytes were exposed for $36 \mathrm{~h}$ to cyclosporin $A(2 \mu \mathrm{g} / \mathrm{ml})$, indomethacin $(20 \mu M)$, nordihydroguaiaretic acid (NDGA; $\left.10^{-6} \mathrm{M}\right)$, dexamethasone $\left(2.5 \times 10^{-7} \mathrm{M}\right)$, or medium $(100 \%)$ conditioned for $36 \mathrm{~h}$ in homologous mononuclear cell cultures (MQ-SN). Values are the mean percentage ( \pm SD) of cells from quadruplicate wells that eliminated ingested bacteria during $8 \mathrm{~h}$ of incubation after challenge (for methods see figure 6). proliferation [10]. As with interferon- $\gamma$ the anticonidial activity of dexamethasone-treated cells is not restored by proliferating lymphocytes. Since pharmacologic concentrations of dexamethasone fail to suppress the activation of antilisterial activity of macrophages by proliferating lymphocytes in mixedcell systems but do not correct the dexamethasoneinduced defect of anticonidial activity, we conclude that in this in vitro system the effector cell in cellmediated immunity is more susceptible to the immunosuppressive effect of glucocorticoids than is the afferent limb, i.e., the proliferating lymphocytes secreting lymphokines. It is of note, however, that this in vitro model represents only incompletely the functions required for a successful immune response and omits important aspects such as the mobilization of $\mathrm{T}$ cells to the site of infection, which may be impaired by glucocorticoid in vivo [7].

\section{Mechanisms of Glucocorticoid-Induced Impairment of the Antimicrobial Activity of Macrophages}

Impairment of the antimicrobial activity of mononuclear phagocytes by glucocorticoids appears to be mediated by the glucocorticoid receptor, since the dose-response curves for the functional defect parallels the dissociation curve for a given glucocorticoid and receptor. Furthermore, the effect is antagonized in a dose-dependent manner by progesterone, an antagonist at the hormone receptor [10]. Glucocorticoids have been shown to alter the metabolism of arachidonic acid in vitro, suppressing the synthesis of prostaglandins and leukotrienes [22]. A comparison of the effects of dexamethasone with those of indomethacin, an inhibitor of prostaglandin synthesis, and nordihydroguaiaretic acid, a nonspecific lipoxygenase inhibitor that also affects leukotriene synthesis, does not, however, suggest that the effect of glucocorticoids on antimicrobial activity of macrophages acts by suppression of leukotriene or prostaglandin synthesis (figure 8 ). The results of transfer experiments with supernatants from autologous cultures of monocytes indicate that suppression of the secretion of monokines is not essential for the effect of glucocorticoids on antimicrobial activity (figure 8 ).

In accordance with the findings of previous studies of Blackwood and Pennington [23] in intact guinea pigs, which suggested that glucocorticoids cause defective intracellular killing without affecting pha- 

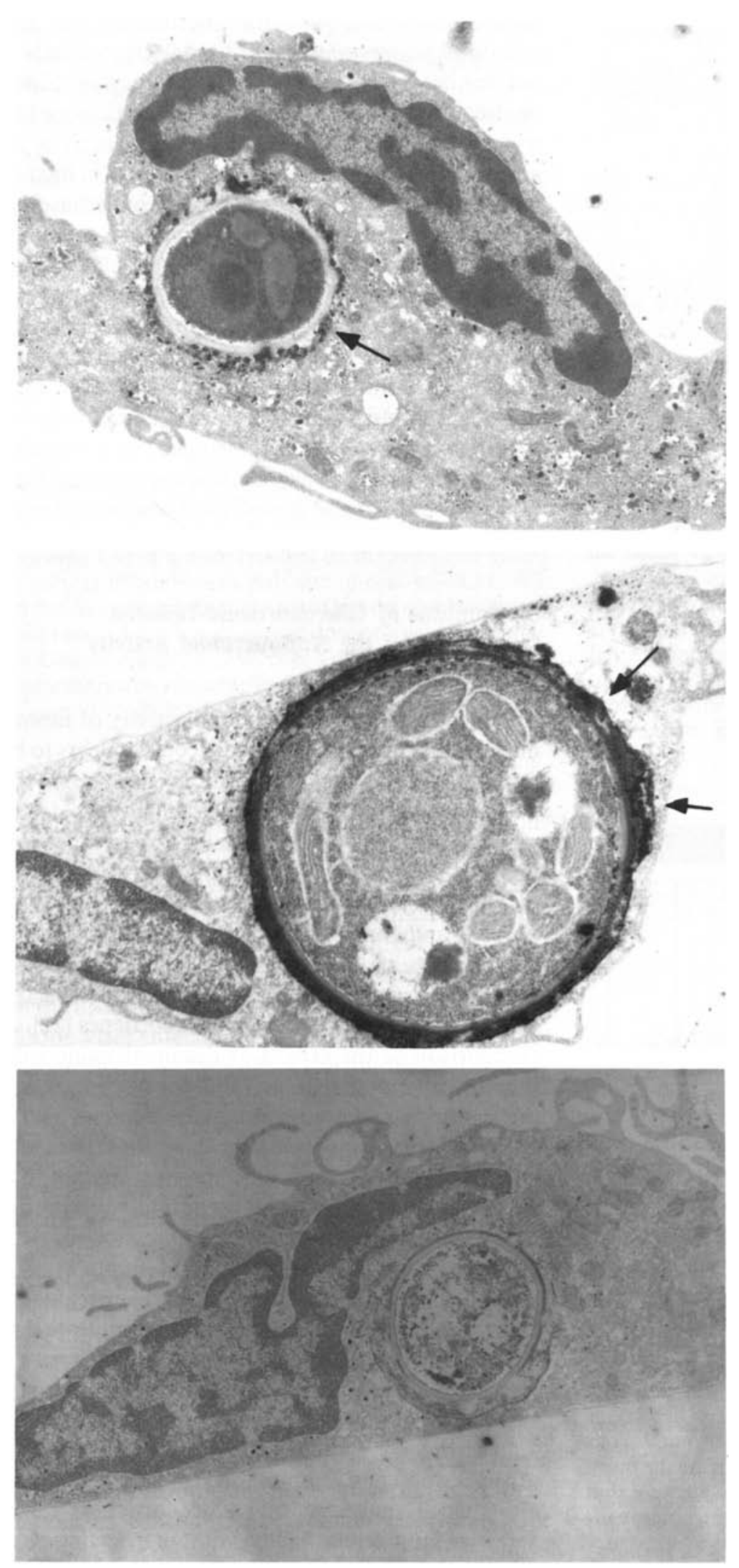

Figure 9. Failure of dexamethasone to prevent fusion of lysosomes with phagosomes containing spores from $A s-$ pergillus fumigatus in human blood-derived macrophages. Human blood-derived macrophages were exposed for 36 $\mathrm{h}$ to $2.5 \times 10^{-7} \mathrm{M}$ dexamethasone before challenge with spores. Electron micrographs of macrophages $4 \mathrm{~h}$ after phagocytosis show acid phosphatase activity (arrows) in phagosome of a control cell (top) and in a dexamethasone-treated cell (middle); no activity is seen in the substrate control (bottom) (final magnification, $\times 14,000$ ). 
gocytosis, our observations of pure phagocyte systems showed that glucocorticoids directly impair the ability of macrophages to kill ingested microorganisms. Because the anticonidial activity of macrophages is independent of oxidative killing systems $[10,24]$, the target for the glucocorticoid effect at the cellular level appears not to lie in the systems that generate reactive oxygen intermediates. In fact, we found that mononuclear phagocytes from children with chronic granulomatous disease - which are unable to mount a respiratory burst - not only handle spores from Aspergillus as efficiently as do cells from normal volunteers, but are equally affected functionally by glucocorticoids [10]. Furthermore, dexamethasone treatment of human blood-derived macrophages for $36 \mathrm{~h}$ does not affect or only minimally affects the generation of hydrogen peroxide or chemiluminescence in a luminol- or lucigeninamplified system upon stimulation with phorbol myristate acetate (PMA) or opsonized zymosan (authors' unpublished observation). It has been proposed by Merkow et al. that lysosomes in macrophages exposed to cortisone do not fuse with phagosomes harboring conidia from Aspergillus [25]. To date we have no evidence in our in vitro model that phagosome-lysosome fusion is disturbed by dexamethasone in cells unable to confine spores (figure 9). Furthermore, alveolar macrophages from mice treated wtih cortisone and challenged with aerosolized spores of Aspergillus fumigatus show that lysosomes, which were visualized by cytochemical methods [26], fuse readily with phagosomes containing spores (authors' unpublished observation).

Taken together, these observations indicate that macrophages exposed to glucocorticoids ingest microorganisms at a normal rate and that after phagocytosis they secrete the usual reactive oxygen intermediates and exhibit normal fusion of lysosomes and phagosomes. Hence, glucocorticoids apparently affect nonoxidative killing systems, which appear to play an impotant role in natural resistance against a broad range of microorganisms. We postulate that this microbicidal system would be operative in "resting," i.e., nonactivated, macrophages but that its activity would be lost under the influence of glucocorticoids. Possibly glucocorticoids act by suppressing the synthesis of one or several specific proteins of nonoxidative killing systems, the suppressive effects being comparable to those of glucocorticoids on the synthesis of elastase, collagenase, and plasminogen activator that were first noted by Werb [27]. Such a catabolic effect would also explain why macrophages must be exposed for 24-36 h to glucocorticoids before any damage to the cells' antimicrobial activity becomes evident [10].

By activating macrophages, interferon enhances the secretion of reactive oxygen intermediates in response to a phagocytic stimulus [28]. The interferon$\gamma$ corrects for the glucocorticoid-induced impairment of the antilisterial activity of macrophages but cannot correct for such a loss of activity against $A$ spergillus spores or Nocardia [10], two pathogens resistant to oxidative killing by neutrophil granulocytes $[23,29]$. Our hypothesis therefore proposes that oxidative killing systems of glucocorticoid-treated macrophages normally react to interferon- $\gamma$ and are normally primed to secrete reactive oxygen intermediates upon challenge. The damage to hitherto unidentified nonoxidative killing systems in glucocorticoidtreated phagocytes would not, however, be alleviated by lymphokine activation. Indeed, we now have evidence that activation of the capacity to secrete $\mathrm{H}_{2} \mathrm{O}_{2}$ or $\mathrm{O}_{2}{ }^{-}$by interferon- $\gamma$-activated macrophages is not impaired by glucocorticoids and that restoration by interferon- $\gamma$ of the antilisterial activity of macrophages after their exposure to glucocorticoids parallels the activation of systems that generate reactive oxygen intermediates (authors' unpublished observation).

\section{Conclusions}

Resident tissue macrophages deployed in great numbers at critical anatomic sites, such as Kupffer cells and alveolar macrophages, appear to be critical targets for the actions of glucocorticoids that compromise the natural resistance of the host. Therapeutic concentrations of glucocorticoids prevent the destruction of microorganisms ingested by macrophages without interfering with phagocytosis, phagosomelysosome fusion, and/or the secretion of reactive oxygen intermediates. These findings indicate that at the cellular level the glucocorticoid target should be looked for in the nonoxidative antimicrobial armature of the phagocyte and point out that nonoxidative killing systems of resident tissue macrophages play an important role in natural resistance to opportunistic pathogens. Glucocorticoids do not prevent lymphokine-induced activation of the macrophages' oxidative killing system. Lymphokines such as interferon- $\gamma$ thus can restore the microbicidal activity of glucocorticoid-suppressed macrophages against organisms susceptible to reactive oxygen in- 
termediates in vitro and most likely can restore the activity in vivo; hence, "glucocorticoid resistance" induced by "established immunity" to an unrelated pathogen seems to reside in the macrophage and does not require specific $\mathrm{T}$ cell interactions. For other potential pathogens that are more resistant to oxidative killing, such as Aspergillus spores and Nocardia, efficient killing depends on other, glucocorticoid-sensitive, mechanisms whose activities cannot be restored by interferon- $\gamma$. The host's inability to counterbalance the effect of glucocorticoids on these defense mechanisms thus might help explain the peculiar susceptibility of glucocorticoidtreated and hypercortisolemic patients to infection with such opportunistic organisms. The nature of the glucocorticoid-sensitive killing mechanisms remains to be elucidated.

\section{References}

1. Ginzler E, Diamond H, Kaplan D, Weiner M, Schlesinger M, Seleznick M. Computer analysis of factors influencing frequency of infection in systemic lupus erythematosus. Arthritis Rheum 1978;21:37-44

2. Anderson RJ, Schafer LA, Olin DB, Eickhoff TC. Infectious risk factors in the immunosuppressed host. Am J Med 1973;54:453-60

3. Gustafson TL, Schaffner W, Lavely GB, Stratton CW, Johnson HK, Hutcheson RH Jr. Invasive aspergillosis in renal transplant recipients: correlation with corticosteroid therapy. J Infect Dis 1983;148:230-8

4. Graham BS, Tucker WS Jr. Opportunistic infections in endogenous Cushing's syndrome. Ann Intern Med 1984; 101:334-8

5. Peterson RE, Wyngaarden JB, Guerra SL, Brodie BB, Bunim $J J$. The physiological disposition and metabolic fate of hydrocortisone in man. J Clin Invest 1955;34:1779-94

6. Webel ML, Ritts RE Jr, Taswell HF, Donadio JV Jr, Woods JE. Cellular immunity after intravenous administration of methylprednisolone. J Lab Clin Med 1974;83:383-92

7. Fauci AS. Immunosuppressive and anti-inflammatory effects of glucocorticoids. In: Baxter JD, Rousseau GG, eds. Glucococorticoid hormone action. Berlin: Springer-Verlag Publishing Co, 1979:449-65

8. Schaffner A, Douglas $H$, Braude A. Selective protection against conidia by mononuclear and against mycelia by polymorphonuclear phagocytes in resistance to Aspergillus: observations on these two lines of defense in vivo and in vitro with human and mouse phagocytes. $J$ Clin Invest 1982;69:617-31

9. Sidransky H, Verney E. Experimental aspergillosis. Lab Invest 1962;11:1172-83

10. Schaffner A. Therapeutic concentrations of glucocorticoids suppress the antimicrobial activity of human macrophages without impairing their responsiveness to gamma interferon. J Clin Invest 1985;76:1755-64
11. North RJ. The action of cortisone acetate on cell-mediated immunity to infection: suppression of host cell proliferation and alteration of cellular composition of infective foci. J Exp Med 1971;134:1485-1500

12. Lurie MB. Role of adrenal cortex in native resistance to tuberculosis. In: Lurie MB, ed. Resistance to tuberculosis: experimental studies in native and acquired defensive mechanisms. Cambridge, Mass: Harvard University Press, 1964:244-64

13. Rinehart JJ, Balcerzak SP, Sagone AL, LoBuglio AF. Effects of corticosteroids on human monocyte function. J Clin Invest 1974;54:1337-43

14. Rinehart JJ, Sagone AL, Balcerzak SP, Ackerman GA, LoBuglio AF. Effects of corticosteroid therapy on human monocyte function. N Engl J Med 1975;292:236-41

15. Schaffner A, Douglas H, Davis CE. Models of $T$ cell deficiency in listeriosis: the effects of cortisone and cyclosporin $A$ on normal and nude BALB/c mice. J Immunol 1983;131:450-3

16. Dannenberg AM Jr. The antiinflammatory effects of glucocorticosteroids: a brief review of the literature. Inflammation 1979;3:329-43

17. Mackaness GB. Cellular resistance to infection, J Exp Med 1962;116:381-406

18. Stevenson MM, Kongshavn PAL, Skamene E. Genetic linkage of resistance to Listeria monocytogenes with macrophage inflammatory responses. J Immunol 1981;127:402-7

19. Lapay DA, Steinman RM, Nathan CF, Murray HW, Cohn ZA. Liver macrophages in murine listeriosis: cell-mediated immunity is correlated with an influx of macrophages capable of generating reactive oxygen intermediates. J Exp Med 1985;161:1503-12

20. Nash TW, Libby DM, Horwitz MA. Interaction between Legionnaires' disease bacterium (Legionella pneumophila) and human alveolar macrophages: influence of antibody, lymphokines, and hydrocortisone. J Clin Invest 1984; 74:771-82

21. Masur H, Murray HW, Jones TC. Effect of hydrocortisone on macrophage response to lymphokine. Infect Immun 1982;35:709-14

22. Blackwood LL, Pennington JE. Dose-dependent effect of glucocorticosteroids on pulmonary defenses in a steroidresistant host. Am Rev Respir Dis 1982;126:1045-9

23. Blackwell GJ, Carnuccio R, Di Rosa M, Flower RJ, Parente L, Persico P. Macrocortin: a polypeptide causing the antiphospholipase effect of glucocorticoids. Nature 1980; 287:147-9

24. Schaffner A, Douglas H, Braude Al, Davis CE. Killing of Aspergillus spores depends on the anatomical source of the macrophage. Infect Immun 1983;42:1109-15

25. Merkow L, Pardo M, Epstein SM, Verney E, Sidransky H. Lysosomal stability during phagocytosis of Aspergillus flavus spores by alveolar macrophages of cortisone-treated mice. Science 1968;160:79-81

26. Oliver $\mathrm{C}$. Cytochemical localization of acid phosphatase and trimetaphosphatase activities in exocrine acinar cells. $\mathbf{J}$ Histochem Cytochem 1980;28:78-81

27. Werb Z. Biochemical actions of glucocorticoids on macrophages in culture. Specific inhibition of elastase, collagenase, and plasminogen activator secretion and effects 
on other metabolic functions. J Exp Med 1978;147: 1695-1712

28. Nathan CF, Murray HW, Wiebe ME, Rubin BY. Identification of interferon- $\gamma$ as the lymphokine that activates human macrophage oxidative metabolism and antimicrobial activity. J Exp Med 1983;158:670-89
29. Filice GA, Beaman BL, Krick JA, Remington JS. Effects of human neutrophils and monocytes on Nocardia asteroides: failure of killing despite occurrence of the oxidative metabolic burst. J Infect Dis 1980;142:432-8 